\title{
Anisakis (Nematoda: Ascaridoidea) from Indonesia
}

\author{
H. W. Palm ${ }^{1,2, *}$, S. Theisen ${ }^{1, *, * *}$, I. M. Damriyasa ${ }^{2}$, E. S. Kusmintarsih ${ }^{3}$, I. B. M. Oka ${ }^{2}$, \\ E. A. Setyowati ${ }^{3}$, N. A. Suratma ${ }^{2}$, S. Wibowo ${ }^{4}$, S. Kleinertz ${ }^{1}$ \\ ${ }^{1}$ Faculty of Agricultural and Environmental Sciences, University of Rostock, Justus-von-Liebig-Weg 6, 18059 Rostock, Germany \\ ${ }^{2}$ Centre for Studies in Animal Diseases, Faculty of Veterinary Medicine, Udayana University Bali, 80363 Badung Denpasar, \\ Bali, Indonesia \\ ${ }^{3}$ Institute of Parasitology and Entomology, Faculty of Biology, Jenderal Soedirman University, 53122 Purwokerto, Java, Indonesia \\ ${ }^{4}$ Ministry of Marine Affairs and Fisheries, Research and Development Centre for Marine and Fisheries Product Processing \\ and Biotechnology, 10260 Jakarta, Java, Indonesia
}

\begin{abstract}
Despite Indonesia's high marine biodiversity, there is a lack of information regarding fish parasites in Indonesian waters. During a sampling of 136 teleost species from Indonesian waters, 22 of them were infected with larvae of Anisakis Dujardin, 1845, a genus with zoonotic potential. We genetically identified 118 worms, provide a revision of all available sequences of the ITS-1-5.8S-ITS-2 marker from Indonesian Anisakis in GenBank ( $=125)$, and establish 16 new host records. So far, 53 Indonesian teleosts harbour Anisakis spp., 32 of them with known sequence data, increasing the worldwide teleosts with genetically identified Anisakis from 155 to 177. Sequence analyses of this marker in the 243 Anisakis specimens identified 3 Anisakis sp. HC2005 and 39 (16\%) A. typica (sensu stricto). A. berlandi and A. pegreffii are reported for the first time from teleosts in the equatorial region and A. physeteris from the Pacific Ocean. The latter 3 species were exclusively found in the migratory scombrid Auxis rochei. Most common infection sites were the body cavity, with 299 (of 848) worms in the mesenteries surrounding the liver, and 129 unattached. Musculature infection was very low, demonstrating minor risk of anisakiasis for human consumers. A total of 193 worms $(\sim 79 \%)$ had a distinct genotype distinguished from $A$. typica by 4 positions in the ITS-1 region. This genotype is reported since 2008 as 'A. typica', 'sibling', 'Anisakis sp./type 1', 'sp. I', 'sp. 2' or 'sp. II'. To avoid further misleading identification, we hereby apply the subspecific entity Anisakis typica var. indonesiensis until description of the adults becomes available.
\end{abstract}

KEY WORDS: Molecular genotyping - Anisakis typica (s.s.) ' A. berlandi - A. pegreffii · A. physeteris $\cdot$ A. typica var. indonesiensis $\cdot$ Anisakiasis

Resale or republication not permitted without written consent of the publisher

\section{INTRODUCTION}

The fisheries industry with its highly valuable food products is a driver for the future economic development of the maritime nation Indonesia. Consequently, food safety and food security are important to consumers. Marine fishes can be a source for foodborne, parasitic human diseases (zoonoses), primarily when larval helminths are ingested through the consumption of semi-cooked

${ }^{*}$ Authors contributed equally

${ }^{* *}$ Corresponding author: stefan.theisen@uni-rostock.de

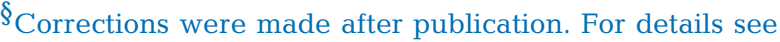
www.int-res.com/articles/dao2017/127/d127p081.pdf

This version: December 19, 2017 or uncooked fisheries products (e.g. Petersen et al. 1993). Some worms can survive inside the human gastrointestinal tract. Especially anisakid nematodes of the genus Anisakis (Anisakidae, Ascaridoidea) have been reported to cause anisakiasis, an inflammation of the human gastrointestinal tract, in many regions worldwide (Ishikura \& Namiki 1989, Ishikura \& Kikuchi 1990, Klimpel \& Palm 2011, Ivanovic et al. 2015, Lim et al. 2015, Sohn et al. 2015, Nieuwenhuizen 2016).

(C) Inter-Research 2017 - www.int-res.com 
Marine fish parasitology in Indonesia is a widely neglected field, and only a few early studies have been published in an international journal. After the first record of the zoonotic nematode genus Anisakis in 1954 (Yamaguti 1954), several Indonesian researchers studied the Anisakidae between 1978 and 2001 based on morphology (Burhanuddin \& Djamali 1978, 1983, Hadidjaja et al. 1978, Hutomo et al. 1978, Ilahude et al. 1978, Ilahude 1980, Martosewojo 1980, Asmanelli et al. 1993, Koesharyani et al. 2001, Lester et al. 2001), adding more host fish species. Recent DNA analyses demonstrated that the genus Anisakis consist of 2 'clades' and different 'complexes'. Clade one includes A. typica (Diesing, 1860), A. nascettii Mattiucci et al., 2009 and its sister species A. ziphidarum Paggi et al., 1998, and 3 siblings forming the A. simplex (sensu lato, s.l.) complex: A. simplex (Rudolphi, 1809) (sensu stricto, s.s.), A. pegreffii Campana-Rouget et Biocca, 1955, and A. berlandi Mattiucci et al., 2014 (formerly known as A. simplex $\mathrm{C}$ of Nascetti et al., 1986). The second clade consists of 3 species forming the A. physeteris (s.1.) complex: A. paggiae Mattiucci et al., 2005, A. brevispiculata Dollfus, 1966, and A. physeteris (Baylis, 1923) (s.s.) (Klimpel \& Palm 2011, Kuhn et al. 2011, 2013, Mattiucci et al. 2005, 2009, 2014). Other genotypes such as 'Anisakis sp. HC-2005' have been rarely reported, lack morphological descriptions, and indicate further taxonomic complexity.

A. typica, the most common species in tropical fishes, has been identified from the southwest Atlantic (Brazil; Nadler et al. 2005), west (Florida; Mattiucci et al. 2005) and east Atlantic (Madeira, Portugal; Pontes et al. 2005), the Mediterranean (North Africa; Farjallah et al. 2008), the central Pacific (Hawaii and Moorea; Kuhn et al. 2013, Palm \& Bray 2014), and most recently from free-living Indo-Pacific bottlenose dolphins Tursiops aduncus off the Hurghada coastline in the northern Red Sea, Egypt (Kleinertz et al. 2014b). Shamsi (2014) summarized the knowledge about Australian Anisakis spp., and identified the species $A$. brevispiculata, A. berlandi, A. pegreffii and A. typica in these waters adjacent to Indonesia, mentioning that $A$. brevispiculata and A. typica show genetic differences to the original genotypes from other areas.

Palm et al. (2008) genetically identified 3 different genotypes of A. typica in Balinese waters. One corresponded with A. typica (s.s.) from GenBank with the same sequence as material from Brazil (from the spinner dolphin Stenella longirostris). The most frequent genotype was distinguishable by 4 positions in the ITS-1 region, a genetic distance similar to the one between the 2 valid species $A$. simplex (s.s.) and A. berlandi (Palm et al. 2008). Since its first record, this genotype was also reported by Koinari et al. (2013), Kuhn et al. (2013), Anshary et al. (2014) and Kleinertz et al. (2014a), under the names A. typica, Anisakis sp. 1, 2 or I and II. The third detected genotype was scarce, and differed in $2 \mathrm{bp}$ between $A$. typica (s.s.) and the Indonesian sibling.

Palm et al. (2008) provided the first infection rates and organ preferences within the fish hosts as well as a comparison of infections in north Javanese and north and south Balinese waters. Based on no muscular infection observed, the authors suggested a minor risk of Anisakis-borne zoonoses in Indonesia. The single published case of anisakiasis in the adjacent Philippines is a wrong citation (cf. Lymbery \& Cheah 2007 with Petersen et al. 1993), but Uga et al. (1996) reported $11 \%$ of 244 tested East Javanese citizens, especially juveniles, positive for antibodies for Anisakis spp. in a seroepidemiological survey. The purpose of the present study was to (1) molecularly genotype the ITS-1-5.8S-ITS-2 region of Anisakis spp. in Indonesia throughout a high number of teleost hosts, (2) analyse the site- and host-specificity of the recorded nematodes and (3) estimate the human health risks caused by Anisakis spp. from Java and Bali. We hereby apply the subspecific entity A. typica var. indonesiensis for the genotype so far solely reported from Indonesia in order to avoid future misidentification.

\section{MATERIALS AND METHODS}

\section{Parasitological examination}

A total of 1801 fishes belonging to 136 species were studied from July 2010 to August 2013. A total of 1531 individuals belonged to 40 teleost species, collected in south Java (Cilacap) and south Bali, Kedonganan (mean of 39 investigated fishes per sample). One species was sampled off north Java (Karimunjawa National Park). According to the salesmen in Cilacap, 2 fish species were originally obtained off Jakarta, northern Java coast. Another sample (270 specimens of 96 species, mean of 3 animals per species) was studied during the First Educational Workshop on Marine Fish Parasites in Bali, July-August 2013. These fishes were collected from fish markets in south Bali (Kedonganan), or were caught alive in southern Balinese waters. Fishes were transferred to the laboratories on ice (or in the case of the workshop, alive in barrels with seawater and sufficient 
oxygen supply), and studied at the Institute for Parasitology and Entomology, Jenderal Soedirman University, Java, and the Centre for Studies in Animal Diseases, Udayana University, Bali.

Morphometrical data for each fish species representing the same size class were taken: total and standard length (TL and SL), in mean and range to the nearest $0.1 \mathrm{~cm}$, and total and gutted weight (TW and GW) to the nearest $0.1 \mathrm{~g}$. The body cavity was opened and studied by naked eye. Internal organs were transferred to Petri dishes with $\mathrm{NaCl}$ solution $(0.9 \%)$, and studied for parasites under a Zeiss Stemi DV4 binocular microscope. The musculature was sliced into $0.5 \mathrm{~cm}$ thin layers and pressed between a set of glass Petri dishes and then studied against a trans-illuminating light source, allowing isolation of anisakids as well as other flesh parasites.

Parasites were pre-identified under a Novel XSZ107BN microscope to separate anisakid nematodes; these were transferred to EtOH 99.6\% for molecular DNA analysis. Specimens belonging to the genus Anisakis are easily distinguished from other nematodes by the distinct ventricle between the oesophagus and intestine, and lack of appendages such as an anterior-directed caecum and posterior-directed appendix (Anderson 2000, Anderson et al. 2009). Ecological parameters for fish infected with Anisakis spp. (prevalence, intensity, abundance) were calculated following Bush et al. (1997).

\section{DNA isolation, PCR and sequencing}

A total of 118 newly collected specimens of Anisakis from 22 teleost species from Javanese and Balinese fish markets were used for molecular identification. Genomic DNA was isolated and purified from individual larvae by using a genomic DNA extraction kit (Peqlab Biotechnology) according to the instructions of the manufacturer. The ribosomal DNA (rDNA) region comprising the ITS-1, 5.8S, ITS-2 and flanking sequences (= ITS + ) were amplified by using the previously described primers NC2 (5'-TTA GTT TCT TTT CCT CCG CT-3') and F2662 ( TK1) (5'-GGC AAA AGT CGT AAC AAG GT-3') (Zhu et al. 2000, Ishiwata et al. 2004, Kuhn et al. 2011). PCR reactions (50 $\mu \mathrm{l})$ included $39 \mu$ l Master-Mix (Peqlab Biotechnology), $3 \mu \mathrm{l}$ of each primer $\left(10 \mathrm{pmol} \mu \mathrm{l}^{-1}\right)$, and $5 \mu \mathrm{l}$ genomic DNA. The Master-Mix contained $5 \mu$ ready mixed Peqlab reaction buffer Y $(4 \mathrm{mM} \mathrm{MgCl} 2,40 \mathrm{mM}$ Tris- $\mathrm{HCl}[\mathrm{pH}$ 8.55], $32 \mathrm{mM}\left(\mathrm{NH}_{4}\right)_{2} \mathrm{SO}_{4}, 0.02 \%$ Tween 20); $0.25 \mu \mathrm{l}$ (2.5 U) Taq polymerase, $1 \mu \mathrm{l}(0.4 \mathrm{mM}) \mathrm{dNTP}$ and 32.75 $\mu l$ water. Each PCR reaction was performed in a ther- mocycler (Biozym Scientific) under the following conditions: initial denaturation at $95^{\circ} \mathrm{C}$ for $1 \mathrm{~min}$; 40 cycles of $94^{\circ} \mathrm{C}$ for $45 \mathrm{~s}$ (denaturation), $55^{\circ} \mathrm{C}$ for $45 \mathrm{~s}$ (annealing), and $72^{\circ} \mathrm{C}$ for $45 \mathrm{~s}$ (extension); followed by a final extension at $72^{\circ} \mathrm{C}$ for $10 \mathrm{~min}$. Control samples without DNA were included in each PCR run. PCR products were examined on $1 \%$ agarose gels. A $100 \mathrm{bp}$ ladder marker (Peqlab Biotechnology) was used to estimate the size of the PCR products. To identify the anisakid nematodes, PCR products were purified with the Cycle-Pure Kit (Peqlab Biotechnology). Afterwards, a total volume of $7 \mu l$, including $2 \mu l$ primer (individually) and $5 \mu \mathrm{l}$ of the PCR product ( 250 ng $\mathrm{\mu l}^{-1}$ ), were sequenced by Seqlab, Göttingen. Both spacers and the 5.8S gene from each PCR product were sequenced, using the primers TK1 and NC2.

\section{Alignment}

Sequences (forward and reverse) of the ITS-1, 5.8S and ITS-2 region were assembled and edited using the BioEdit sequence alignment editor (v. 7.1.3.0). They were compared manually with the original chromatograms, identified via GenBank and aligned with a previously characterized sequence (GenBank) data of Ansiakis spp., and with 125 previously identified Anisakis sequences from Indonesia (see Palm et al. 2008, Koinari et al. 2013, Kuhn et al. 2013, Anshary et al. 2014, Kleinertz et al. 2014a) using ClustalW (v. 1.83) multiple sequence alignments (settings: full multiple alignment, gap penalties default) (Thompson et al. 1994). They were then aligned with sequences from other Anisakis species from GenBank for species and genotype identification as follows: $A$. typica (s.s.) from the spinner dolphin Stenella longirostris from Brazil (AY826724; see Nadler et al. 2005), A. simplex C, now A. berlandi from Mirounga angustirostris from California (AY821739; see Nadler et al. 2005), A. pegreffii and A. physeteris from mackerels from Japan (AB277823 and AB277821 respectively; see Umehara et al. 2008), and Anisakis sp. HC-2005 from Hoplostethus cadenati from the African shelf (EU718474; see Kijewska et al. 2009). We hereby apply the subspecific entity Anisakis typica var. indonesiensis for most of the specimens recorded during the present study, and for the specimens from Auxis rochei from Indonesia (EU346093; see Palm et al. 2008). Because we do not nominate an available name but apply the subspecific entity, we must not follow the ICZN (2016, their §1.3). New nucleotide sequence data are available in GenBank (accession numbers KY524195-KY524217). 


\section{RESULTS}

\section{Parasite infection}

A total of 6 different genotypes of Anisakis were identified, including Anisakis sp. HC-2005, A. berlandi, A. pegreffii, A. physeteris, A. typica (s.s.), and an Indonesian $A$. typica genotype for which we hereby apply the subspecific entity $A$. typica var. indonesiensis. This allows distinction of all thus far analysed A. typica from Indonesian waters, formerly named A. typica, Anisakis sp., sp. 1, sp. 2, I and II into 2 distinct genotypes. A. berlandi and $A$. pegreffii are hereby reported for the first time from teleost hosts from a tropical locality, and A. physeteris from the Pacific Ocean. During the detailed parasite sampling with at least 20-75 fish specimens per sample (39 in general), 16 of 40 teleost hosts were found to be infected with Anisakis spp. in Indonesian waters. Six fish species were found to be infected during the smaller samplings at the Bali workshop in 2013. The infected fish species are given in Tables $1 \& 2$. The Anisakis-negative fish species of the former, detailed sampling, mainly from Cilacap, south Central Java (other origins are mentioned), were Caesionidae: Caesio cuning $(\mathrm{n}=35$, mean TL $[\mathrm{mTL}]=21.7 \mathrm{~cm})$, Pterocaesio diagramma $(\mathrm{n}=20$, $\mathrm{mTL}=21.9 \mathrm{~cm})$ (both from Kedonganan); Carangidae: Alepes melanoptera $(\mathrm{n}=20, \mathrm{mTL}=23.0 \mathrm{~cm})$, Atropus atropos $(\mathrm{n}=35, \mathrm{mTL}=16.4 \mathrm{~cm})$, Atule mate $(\mathrm{n}=75, \mathrm{mTL}=23.4 \mathrm{~cm})(35$ from Kedonganan), Caranx heberi $(\mathrm{n}=35, \mathrm{mTL}=21.2 \mathrm{~cm})$, Parastromateus niger $(\mathrm{n}=40, \mathrm{mTL}=19.4 \mathrm{~cm})$, Selar boops $(\mathrm{n}=37, \mathrm{mTL}=17.5 \mathrm{~cm})$; Cichlidae: Oreochromis mossambicus $(\mathrm{n}=35, \mathrm{mTL}=16.9 \mathrm{~cm})$; Clupeidae: Amblygaster sirm $(\mathrm{n}=35, \mathrm{mTL}=18.9$ $\mathrm{cm})$, Nematalosa come $(\mathrm{n}=35, \mathrm{mTL}=21.8 \mathrm{~cm})$, Sardinella lemuru $(\mathrm{n}=35, \mathrm{mTL}=19.4 \mathrm{~cm})$ (the latter 2 from Kedonganan); Cynoglossidae: Cynoglossus $\mathrm{cf}$. arel $(\mathrm{n}=35, \mathrm{mTL}=27.6 \mathrm{~cm})$; Haemulidae: Pomadasys kaakan $(\mathrm{n}=35, \mathrm{mTL}=19.7 \mathrm{~cm})$; Lutjanidae: Lutjanus vitta $(\mathrm{n}=35, \mathrm{mTL}=20.1 \mathrm{~cm}$; however, the low sample survey from the workshop was infected); Nemipteridae: Nemipterus japonicus $(\mathrm{n}=35, \mathrm{mTL}=20.6 \mathrm{~cm}) ;$ Sciaenidae: Nibea soldado $(\mathrm{n}=35, \mathrm{mTL}=23.3 \mathrm{~cm})$, Otolithes ruber $(\mathrm{n}=30, \mathrm{mTL}=17.6 \mathrm{~cm})$, Pennahia macrocephalus $(\mathrm{n}=40, \mathrm{mTL}=17.6 \mathrm{~cm}) ;$ Scombridae: Rastrelliger kanagurta $(\mathrm{n}=35, \mathrm{mTL}=23.8 \mathrm{~cm})$, Sarda orientalis $(\mathrm{n}=35, \mathrm{mTL}=29.3 \mathrm{~cm})$ (Kedonganan); Epinephelidae: Epinephelus fuscoguttatus (aquaculture, $\mathrm{n}=75, \mathrm{mTL}=26.0 \mathrm{~cm}$ ); Sparidae: Acan- thopagrus berda $(\mathrm{n}=35, \mathrm{mTL}=21.6 \mathrm{~cm})$; and Stromateidae: Pampus argenteus $(\mathrm{n}=35, \mathrm{mTL}=$ $22.0 \mathrm{~cm}$ ).

A total of 22 fish species were found to be infected with Anisakis spp., and 16 new host records could be established (Table 2). Revision of 125 available sequence data allowed confirmation of 53 Anisakisinfected fish host species from Indonesia, including 32 with genetically confirmed identity (Tables $3 \& 4$ ). Table 2 presents the infection rates (prevalence, intensity, abundance) within the macrohabitat ( fish host species) and the site of infection (microhabitat). Because some data go back to earlier studies that allow no molecular identification without further sampling, Table 2 refers in these cases to 'Anisakis spp.'

Highest infection rates were documented for the scombrid Auxis rochei and the carangid Selar crumenophthalmus (prevalence of $97.2 \%$ and $81.1 \%$ respectively). In both fish species, the range of microhabitats was widest (Table 2). We isolated 848 worms, almost all from the body cavity, free or attached to the outer tissues of various organs and/or the mesenteries, with just a single exception: One worm was isolated from the muscle tissue (filet) of a $S$. crumenophthalmus. The preferred sites for attachment were the liver $(\mathrm{n}=299)$, gonads $(\mathrm{n}=$ 138), stomach $(\mathrm{n}=136)$, body cavity (freely) ( $\mathrm{n}=$ 131), intestine $(n=95)$, pyloric caeca $(n=45)$. The swim bladder, gill cavity, fins and muscle tissue (filet) were generally uninfected $(\mathrm{n}=1$ for each of the latter). Infections in gills and fins are unusual for this endoparasite and probably the effect of e.g. everted stomachs or a post-mortem migration (Table 2). The selected size range of the studied fish of a respective species was similar, preventing detailed comparison of different size classes. Male and female fishes had infection levels as follows: (1) Auxis rochei: 135 worms were isolated from 12 males, while 532 worms originated from 24 females; (2) Selar crumenophthalmus: 18 males harboured 44 worms while 7 females harboured 19 Anisakis; (3) Terapon jarbua: 8 worms from 17 males and 9 worms from 18 females; and (4) Priacanthus tayenus: 7 worms were obtained from 24 males and 8 worms from 11 females. A total of 797 of the 848 worms $(\sim 94 \%)$ were sampled during the dry season, with the remaining 51 specimens $(\sim 6 \%)$ during the rainy season. A total of 804 worms $(\sim 95 \%)$ originated from Kedonganan, Bali, and 44 ( $5 \%)$ from Cilacap, south Java (although similar fish quantities were analysed from rainy vs. dry seasons respectively in Cilacap vs. Kedonganan; cf. Tables 1 \& 2). 
Table 1. Fish samples infected by Anisakis spp., with locality, season, number of sampled individuals ( $\left.\mathrm{n}_{\text {fish }}\right)$, total length (TL), total weight (TW), gutted weight (GW) in mean and range, and number of males (M), females (F) and juveniles (J). Additional fish species samples hosting Anisakis (workshop, dry season 2013): Brama cf. orcini (Bramidae) $(\mathrm{n}=1)$, Epinephelus areolatus (Epinephelidae) $(\mathrm{n}=3)$, E. longispinis (Epinephelidae) $(\mathrm{n}=1)$, Lutjanus argentimaculatus (Lutjanidae) $(\mathrm{n}=1)$, L. vitta $($ Lutjanidae) $(\mathrm{n}=3)$, and Sufflamen fraenatum (Balistidae) $(\mathrm{n}=2)$

\begin{tabular}{|c|c|c|c|c|c|c|c|c|c|}
\hline Fish species and family & Area & Season, year & $\mathrm{n}_{\text {fish }}$ & $\mathrm{TL}(\mathrm{cm})$ & TW (g) & GW (g) & M & $\mathrm{F}$ & $\mathrm{J}$ \\
\hline $\begin{array}{l}\text { Auxis rochei } \\
\text { (Scombridae) }\end{array}$ & Kedonganan & Dry, 2010 & 36 & $\begin{array}{c}30.7 \\
(27.6-33.5)\end{array}$ & $\begin{array}{c}357.9 \\
(240.2-459.9)\end{array}$ & $\begin{array}{c}295.5 \\
(191.3-393.8)\end{array}$ & 12 & 24 & - \\
\hline $\begin{array}{l}\text { A. thazard } \\
\text { (Scombridae) }\end{array}$ & Kedonganan & Dry, 2010 & 35 & $\begin{array}{c}28.3 \\
(25.7-31.5)\end{array}$ & $\begin{array}{c}269.8 \\
(201.2-384.5)\end{array}$ & $\begin{array}{c}232.2 \\
(167.3-303.3)\end{array}$ & - & - & 35 \\
\hline $\begin{array}{l}\text { Decapterus macrosoma } \\
\text { (Carangidae) }\end{array}$ & Kedonganan & Rainy, 2011 & 35 & $\begin{array}{c}20.5 \\
(19.1-22.5)\end{array}$ & $\begin{array}{c}76.2 \\
(61.4-98.8)\end{array}$ & $\begin{array}{c}66.3 \\
(50.7-87.3)\end{array}$ & 14 & 21 & - \\
\hline $\begin{array}{l}\text { D. tabl } \\
\text { (Carangidae) }\end{array}$ & Kedonganan & Dry, 2010 & 35 & $\begin{array}{c}18.0 \\
(17.1-19.1)\end{array}$ & $\begin{array}{c}50.3 \\
(43.2-58.7)\end{array}$ & $\begin{array}{c}45.6 \\
(39.3-53.0)\end{array}$ & - & - & 35 \\
\hline $\begin{array}{c}\text { Epinephelus ongus } \\
\text { (Epinephelidae) }\end{array}$ & Karimunjawa & Rainy, 2013 & 35 & $\begin{array}{c}25.5 \\
(20.5-30.4)\end{array}$ & $\begin{array}{c}266.3 \\
(108.5-498.0)\end{array}$ & $\begin{array}{c}238.0 \\
(99.8-463.6)\end{array}$ & 7 & 23 & 5 \\
\hline $\begin{array}{l}\text { E. sexfasciatus } \\
\text { (Epinephelidae) }\end{array}$ & Jakarta & Rainy, 2012 & $41^{\mathrm{a}}$ & $\begin{array}{c}25.1 \\
(23.1-27.8)\end{array}$ & $\begin{array}{c}240.5 \\
(189.0-352.4)\end{array}$ & $\begin{array}{c}225.1 \\
(176.6-325.9)\end{array}$ & - & 41 & - \\
\hline $\begin{array}{l}\text { Megalaspis cordyla } \\
\text { (Carangidae) }\end{array}$ & Kedonganan & Dry, 2010 & $35^{\mathrm{b}}$ & $\begin{array}{c}32.5 \\
(29.5-36.0)\end{array}$ & $\begin{array}{c}303.9 \\
(246.3-403.7)\end{array}$ & $\begin{array}{c}282.0 \\
(226.7-373.4)\end{array}$ & 20 & 15 & - \\
\hline $\begin{array}{l}\text { Nemipterus furcosus } \\
\text { (Nemipteridae) }\end{array}$ & Kedonganan & Dry, 2010 & 35 & $\begin{array}{c}24.4 \\
(22.7-26.6)\end{array}$ & $\begin{array}{c}165.8 \\
(136.1-231.1)\end{array}$ & $\begin{array}{c}151.3 \\
(119.5-210.7)\end{array}$ & 11 & 24 & - \\
\hline $\begin{array}{l}\text { Opisthopterus tardoore } \\
\text { (Pristigasteridae) }\end{array}$ & Cilacap & Rainy, 2011 & 35 & $\begin{array}{c}21.0 \\
(18.6-23.7)\end{array}$ & $\begin{array}{c}52.9 \\
(36.6-68.1)\end{array}$ & $\begin{array}{c}50.1 \\
(35.2-63.5)\end{array}$ & 9 & 26 & - \\
\hline $\begin{array}{l}\text { Pomadasys maculatus } \\
\text { (Haemulidae) }\end{array}$ & Cilacap & Rainy, 2012 & 35 & $\begin{array}{c}18.2 \\
(16.5-22.0)\end{array}$ & $\begin{array}{c}104.3 \\
(71.6-194.5)\end{array}$ & $\begin{array}{c}94.4 \\
(62.0-169.5)\end{array}$ & 18 & 17 & - \\
\hline $\begin{array}{l}\text { Priacanthus tayenus } \\
\text { (Priacanthidae) }\end{array}$ & Cilacap & Rainy, 2011 & 35 & $\begin{array}{c}26.3 \\
(21.7-31.2)\end{array}$ & $\begin{array}{c}177.8 \\
(137.0-238.6)\end{array}$ & $\begin{array}{c}160.6 \\
(121.0-213.7)\end{array}$ & 24 & 11 & - \\
\hline $\begin{array}{l}\text { Scomberoides tol } \\
\text { (Carangidae) }\end{array}$ & Cilacap & Rainy, 2012 & 35 & $\begin{array}{c}27.6 \\
(24.0-31.0)\end{array}$ & $\begin{array}{c}138.4 \\
(90.0-207.5)\end{array}$ & $\begin{array}{c}120.3 \\
(80.7-170.0)\end{array}$ & 9 & 26 & - \\
\hline $\begin{array}{l}\text { Selar crumenophthalmus } \\
\quad \text { (Carangidae) }\end{array}$ & Kedonganan & Dry, 2010 & $37^{c}$ & $\begin{array}{c}18.34 \\
(15.5-20.0)\end{array}$ & $\begin{array}{c}72.5 \\
(46.2-93.4)\end{array}$ & $\begin{array}{c}61.8 \\
(38.5-81.0)\end{array}$ & 18 & 7 & 12 \\
\hline $\begin{array}{l}\text { Selaroides leptolepis } \\
\text { (Carangidae) }\end{array}$ & Cilacap & Dry, 2011 & $25^{\mathrm{d}}$ & $\begin{array}{c}15.1 \\
(14.0-16.8)\end{array}$ & $\begin{array}{c}38.5 \\
(31.6-50.3)\end{array}$ & $\begin{array}{c}36.4 \\
(29.9-49.0)\end{array}$ & 15 & 10 & - \\
\hline $\begin{array}{l}\text { Terapon jarbua } \\
\text { (Terapontidae) }\end{array}$ & Cilacap & Rainy, 2011 & 35 & $\begin{array}{c}24.2 \\
(20.3-28.9)\end{array}$ & $\begin{array}{c}201.7 \\
(107.7-310.0)\end{array}$ & $\begin{array}{c}180.6 \\
(94.8-265.2)\end{array}$ & 17 & 18 & - \\
\hline $\begin{array}{l}\text { Trichiurus lepturus } \\
\text { (Trichiuridae) }\end{array}$ & Kedonganan & Dry, 2010 & 35 & $\begin{array}{c}59.4 \\
(48.2-67.9)\end{array}$ & $\begin{array}{c}154.2 \\
(95.6-225.1)\end{array}$ & $\begin{array}{c}145.1 \\
(87.8-205.1)\end{array}$ & 25 & 10 & - \\
\hline $\begin{array}{l}{ }^{\text {a }} \text { Sample bought in Cilacar } \\
\text { did not harbour any Anis } \\
{ }^{\mathrm{b}} \text { A second sample of } 40 \text { fis } \\
{ }^{\mathrm{c}} \text { A second sample of } 25 \mathrm{fis} \\
{ }^{\mathrm{d}} \text { Additional } 8 \text { specimens } \mathrm{fr}\end{array}$ & $\begin{array}{l}\text { o, but originated } \\
\text { akis } \\
\text { hes from Cilaca } \\
\text { hes from Cilaca] } \\
\text { com Kedonganal }\end{array}$ & $\begin{array}{l}\text { from Jakarta } \\
\text { p did not harbc } \\
\text { p did not harbo } \\
\text { (workshop, d }\end{array}$ & $\begin{array}{l}\text { cordir } \\
\text { r any } \\
\text { Ir any }\end{array}$ & $\begin{array}{l}\text { ng to salesma } \\
\text { Anisakis } \\
\text { Anisakis }\end{array}$ & an. Another sam & ple of 35 fishe: & es fr & $\mathrm{m} \mathrm{Ci}$ & acap \\
\hline
\end{tabular}

\section{Molecular analyses}

The ITS rDNA sequence data of 243 Indonesian larvae of Anisakis spp. (118 from the present study and 125 from previously sequenced helminths, named in the literature as e.g. real, original, 'sensu stricto' typica, 'typica sibling', or Anisakis sp./type 1, sp. 2, sp. I, sp. II) from 32 different bony fishes revealed the presence of 4 valid Anisakis species (Tables 3 \& 4): A. physeteris $(\mathrm{n}=4)$, A. pegreffii $(\mathrm{n}=1), A$. berlandi $(\mathrm{n}=2)$ and A. typica (s.s.) ( $\mathrm{n}=39)$. One further genotype, as defined and illustrated by Palm et al. (2008), was pre- dominantly found. We hereby apply the subspecific entity A. typica var. indonesiensis for these specimens. These 193 ( 79\%) worms showed a 4 bp difference at identical positions in the ITS-1 region to the closely related A. typica (s.s.) (for alignment, see Palm et al. 2008). The genetic distance by using 903 informative bp was the same to the one between 2 valid species, A. simplex (s.s.) and A. berlandi (4 bp). This genotype was isolated from 28 out of 32 fish species with genetically identified Anisakis infection ( 88\%). On the other hand, A. typica (s.s.) (16\% of the analysed worms) was documented in only $28 \%(9 / 32)$ of 
Table 2. Infected fish species with number of Anisakis individuals genetically identified (total $\mathrm{n}_{\text {Anisakis }}$ DNA-analysed $=118$ ) and infection rates, with total number of specimens $\left(\mathrm{n}_{\text {Anisakis }}\right.$ documented), prevalence $(\mathrm{P})$ (proportion of infected hosts among all hosts examined), mean intensity (mI) (mean number of Anisakis found in infected hosts), mean abundance (mA) (mean number of Anisakis found in all hosts), and infection sites (all data from this study) (bcv: body cavity, gcv: gill cavity; go: gonads; in: intestine; li: liver; mus: muscle tissue; pyl: pylorus; st: stomach; swb: swim bladder). Worms were not isolated from inner lumen of organs, but were located outside, attached to organs or mesenteries. Thus, all worms originated from the body cavity (except the one from muscle), sometimes free-living and sometimes attached to organs

\begin{tabular}{|c|c|c|c|c|c|c|}
\hline Fish species and family & $\begin{array}{c}\mathrm{n}_{\text {Anisakis }} \\
\text { documented }\end{array}$ & $\begin{array}{l}\mathrm{P} \\
(\%)\end{array}$ & $\begin{array}{c}\mathrm{mI} \\
\text { (range) }\end{array}$ & $\mathrm{mA}$ & Site of infection & $\begin{array}{c}\mathrm{n}_{\text {Anisakis }} \\
\text { DNA-analysed }\end{array}$ \\
\hline $\begin{array}{l}\text { Auxis rochei } \\
\text { (Scombridae) }\end{array}$ & 667 & 97.2 & $\begin{array}{c}19.1 \\
(1-275)\end{array}$ & 18.53 & $\begin{array}{l}293 \text { (li), } 125 \text { (go), } 115 \text { (st), } \\
64 \text { (in), } 46 \text { (bcv), } 24 \text { (pyl) }\end{array}$ & 24 \\
\hline $\begin{array}{l}\text { A. thazard } \\
\text { (Scombridae) }\end{array}$ & 10 & 22.9 & $\begin{array}{c}1.3 \\
(1-3)\end{array}$ & 0.29 & 7 (bcv), 3 (li) & 10 \\
\hline $\begin{array}{l}\text { Decapterus macrosoma } \\
\text { (Carangidae) }\end{array}$ & 8 & 20.0 & $\begin{array}{c}1.1 \\
(1-2)\end{array}$ & 0.23 & $\begin{array}{c}4 \text { (bcv), } 1 \text { (swb), } 1 \text { (st) } \\
1 \text { (go), } 1 \text { (in) }\end{array}$ & 8 \\
\hline $\begin{array}{l}\text { D. } \text { tabl }^{\mathrm{a}} \\
\quad \text { (Carangidae) }\end{array}$ & 12 & 25.7 & $\begin{array}{c}1.2 \\
(1-2)\end{array}$ & 0.31 & 10 (bcv), 2 (st) & 12 \\
\hline $\begin{array}{l}\text { Epinephelus ongus } \\
\text { (Epinephelidae) }\end{array}$ & 3 & 8.6 & $\begin{array}{l}1.0 \\
(1)\end{array}$ & 0.09 & 1 (go), 1 (in), 1 (pyl) & 1 \\
\hline $\begin{array}{l}\text { E. sexfasciatus } \\
\text { (Epinephelidae) }\end{array}$ & 3 & 8.6 & $\begin{array}{l}1.0 \\
(1)\end{array}$ & 0.09 & 1 (go), 1 (in), 1 (pyl) & 3 \\
\hline $\begin{array}{l}\text { Megalaspis cordyla } \\
\text { (Carangidae) }\end{array}$ & 1 & 2.9 & $\begin{array}{l}1.0 \\
(1)\end{array}$ & 0.03 & 1 (li) & 1 \\
\hline $\begin{array}{l}\text { Nemipterus furcosus } \\
\text { (Nemipteridae) }\end{array}$ & 3 & 5.7 & $\begin{array}{c}1.5 \\
(1-2)\end{array}$ & 0.06 & 2 (go), 1 (st) & 3 \\
\hline $\begin{array}{l}\text { Opisthopterus tardoore } \\
\text { (Pristigasteridae) }\end{array}$ & 2 & 5.7 & $\begin{array}{l}1.0 \\
(1)\end{array}$ & 0.06 & 1 (pyl), 1 (bcv) & 2 \\
\hline $\begin{array}{l}\text { Pomadasys maculatus } \\
\text { (Haemulidae) }\end{array}$ & 1 & 2.9 & $\begin{array}{l}1.0 \\
(1)\end{array}$ & 0.03 & 1 (in) & 1 \\
\hline $\begin{array}{l}\text { Priacanthus tayenus }^{\mathrm{a}} \\
\text { (Priacanthidae) }\end{array}$ & 15 & 34.3 & $\begin{array}{c}1.3 \\
(1-2)\end{array}$ & 0.43 & 13 (in), 1 (pyl), 1 (bcv) & 10 \\
\hline $\begin{array}{l}\text { Scomberoides tol } \\
\text { (Carangidae) }\end{array}$ & 2 & 5.7 & $\begin{array}{l}1.0 \\
(1)\end{array}$ & 0.06 & 1 (go), 1 (bcv) & 2 \\
\hline $\begin{array}{l}\text { Selar crumenophthalmus } \\
\text { (Carangidae) }\end{array}$ & 91 & 81.1 & $\begin{array}{c}3.0 \\
(1-8)\end{array}$ & 2.46 & $\begin{array}{c}49 \text { (bcv), } 15 \text { (pyl), } 11 \text { (st), } 7 \text { (in), } \\
6 \text { (go), } 1 \text { (gcv), } 1 \text { (fin), } 1 \text { (mus) }\end{array}$ & 16 \\
\hline $\begin{array}{l}\text { Selaroides leptolepis }{ }^{\mathrm{a}} \\
\text { (Carangidae) }\end{array}$ & 1 & 4.0 & $\begin{array}{l}1.0 \\
(1)\end{array}$ & 0.04 & 1 (go) & 1 \\
\hline $\begin{array}{l}\text { Terapon jarbua } \\
\text { (Terapontidae) }\end{array}$ & 17 & 37.1 & $\begin{array}{c}1.3 \\
(1-3)\end{array}$ & 0.49 & 6 (st), 5 (in), 2 (pyl), 2 (li), 2 (bcv) & 12 \\
\hline $\begin{array}{l}\text { Trichiurus lepturus } \\
\text { (Trichiuridae) }\end{array}$ & 1 & 2.9 & $\begin{array}{l}1.0 \\
(1)\end{array}$ & 0.03 & 1 (in) & 1 \\
\hline \multicolumn{7}{|c|}{ Additional Anisakis-hosting fish species investigated during low-sample spot survey (workshop 2013): } \\
\hline $\begin{array}{l}\text { Brama cf. orcini }{ }^{\text {a }} \\
\text { (Bramidae) }\end{array}$ & 1 & & & & 1 (in) & 1 \\
\hline $\begin{array}{l}\text { E. areolatus } \\
\text { (Epinephelidae) }\end{array}$ & 5 & & & & $5(\mathrm{bcv})$ & 5 \\
\hline $\begin{array}{l}\text { E. longispinis }{ }^{\mathrm{a}} \\
\text { (Epinephelidae) }\end{array}$ & 1 & & & & 1 (bcv) & 1 \\
\hline $\begin{array}{l}\text { Lutjanus argentimaculatus }{ }^{\mathrm{a}} \\
\text { (Lutjanidae) }\end{array}$ & 1 & & & & 1 (bcv) & 1 \\
\hline $\begin{array}{l}\text { L. vitta } \\
\quad \text { (Lutjanidae) }\end{array}$ & 2 & & & & $2(\mathrm{bcv})$ & 2 \\
\hline $\begin{array}{l}\text { Sufflamen fraenatum }{ }^{\mathrm{a}} \\
\text { (Balistidae) }\end{array}$ & 1 & & & & 1 (bcv) & 1 \\
\hline
\end{tabular}


Table 3. Host records of Anisakis spp. from Indonesia. Anisakis typica var. indonesiensis (A. t. indonesiensis) represents the local genotype

\begin{tabular}{|c|c|c|c|c|}
\hline $\begin{array}{l}\text { Fish host: } \\
\text { family and species }\end{array}$ & Parasite & Locality & Reference & $\begin{array}{l}\text { DNA } \\
\text { quenced }\end{array}$ \\
\hline \multicolumn{5}{|l|}{ Ariommatidae } \\
\hline $\begin{array}{l}\text { Ariomma indicum } \\
(=A \text {. indica })^{\mathrm{a}}\end{array}$ & Anisakis sp. & S. Java & Theisen (2009) & \\
\hline \multicolumn{5}{|l|}{ Balistidae } \\
\hline Sufflamen fraenatum & Anisakis sp. HC-2005 & Bali & Present study & Yes \\
\hline \multicolumn{5}{|l|}{ Bramidae } \\
\hline Brama dussumieri & Anisakis sp. & S. Java & Jakob \& Palm (2006) & \\
\hline Brama cf. orcini & A. $t$. indonesiensis & Bali & Present study & Yes \\
\hline \multicolumn{5}{|l|}{ Caesionidae } \\
\hline Caesio cuning & A. t. indonesiensis & Bali, 'Indonesia' & Palm et al. (2008), Kuhn et al. (2013) & Yes \\
\hline Caesio sp. & Anisakis sp. & S. Java & Setyobudi et al. (2011) & \\
\hline \multicolumn{5}{|l|}{ Carangidae } \\
\hline Atule mate & Anisakis sp. & N. Java & \multicolumn{2}{|l|}{ Ilahude et al. (1978), Ilahude (1980) } \\
\hline Caranx sp. & A. typica (s.l.) $)^{\mathrm{b}}$ & Makassar Strait & \multicolumn{2}{|l|}{$\begin{array}{l}\text { Anshary et al. (2014), } \\
\text { Anshary pers. comm. (Feb 2015) }\end{array}$} \\
\hline Decapterus kurroides & Anisakidae & N. Java & \multicolumn{2}{|l|}{ Burhanuddin \& Djamali (1983) } \\
\hline D. macarellus & A. t. indonesiensis & Papua New Guinea & Koinari et al. (2013) & Yes \\
\hline D. macrosoma & A. $t$. indonesiensis & Bali & Present study & Yes \\
\hline D. russelli $(=D \text {. lajang })^{\mathrm{a}}$ & Anisakis sp. & Bali, Java & \multicolumn{2}{|c|}{$\begin{array}{l}\text { Burhanuddin \& Djamali (1978, 1983), } \\
\text { Hadidjaja et al. (1978), Hutomo et al. (1978), } \\
\text { Martosewojo (1980), Palm et al. (2008) }\end{array}$} \\
\hline \multirow[t]{2}{*}{ D. tabl } & A. typica (s.s.) & Bali & Present study & Yes \\
\hline & A. $t$. indonesiensis & Bali & Present study & Yes \\
\hline Megalaspis cordyla & A. $t$. indonesiensis & Bali & Present study & Yes \\
\hline Scomberoides tol & A. $t$. indonesiensis & S. Java & Present study & Yes \\
\hline \multirow{2}{*}{$\begin{array}{l}\text { Selar crumenophthalmus } \\
\text { (= Caranx }\end{array}$} & Anisakis sp. & N. Java & \multicolumn{2}{|l|}{ Ilahude et al. (1978), Ilahude (1980) } \\
\hline & A. typica (s.s.) & Papua New Guinea, Bali & Koinari et al. (2013), present study & Yes \\
\hline crumenophthalmus) $^{\mathrm{a}}$ & A. $t$. indonesiensis & Bali & Present study & Yes \\
\hline Selaroides leptolepis & A. t. indonesiensis & S. Java & Present study & Yes \\
\hline \multicolumn{5}{|l|}{ Clupeidae } \\
\hline Amblygaster sirm & Anisakis sp. & N. Java, Sunda Strait & \multicolumn{2}{|l|}{$\begin{array}{c}\text { et al. (1978), Martosewojo (1980), } \\
\text { Burhanuddin \& Djamali (1983) }\end{array}$} \\
\hline Sardinella fimbriata & Anisakidae & N. Java, Sunda Strait & \multicolumn{2}{|l|}{ Burhanuddin \& Djamali (1983) } \\
\hline S. gibbosa $(=\text { jussieui })^{\mathrm{a}}$ & Anisakis sp. & N. Java, Sunda Strait & \multicolumn{2}{|l|}{ Ilahude et al. (1978), Ilahude (1980) } \\
\hline \multicolumn{5}{|l|}{ Coryphaenidae } \\
\hline \multirow[t]{2}{*}{ Coryphaena hippurus } & A. typica (s.s.) & S. Java & Palm et al. (2008) & Yes \\
\hline & A. $t$. indonesiensis & S. Java & Palm et al. (2008) & Yes \\
\hline \multicolumn{5}{|l|}{ Epinephelidae } \\
\hline $\begin{array}{c}\text { Cephalopholis } \\
\text { cyanostigma }\end{array}$ & A. typica (s.l.) $)^{\mathrm{b}}$ & Makassar Strait & \multicolumn{2}{|l|}{$\begin{array}{c}\text { Anshary et al. (2014), } \\
\text { H. Anshary (pers. comm.) }\end{array}$} \\
\hline \multirow{3}{*}{$\begin{array}{l}\text { Cromileptes altivelis } \\
\text { Epinephelus areolatus }\end{array}$} & Anisakidae & Bali & \multicolumn{2}{|l|}{ Koesharyani et al. (2001) } \\
\hline & A. t. indonesiensis & S. Java, Bali & $\begin{array}{l}\text { Palm et al. (2008), Kleinertz et al. } \\
\text { (2014a), present study }\end{array}$ & Yes \\
\hline & Anisakis sp. HC-2005 & S. Java & Kleinertz et al. (2014a) & Yes \\
\hline E. fuscoguttatus & Anisakis sp. & N. Java, Sulawesi & $\begin{array}{c}\text { Burhanuddin \& Djamali (1983), } \\
\text { Asmanelli et al. (1993) }\end{array}$ & \\
\hline E. longispinis & A. t. indonesiensis & Bali & Present study & Yes \\
\hline E. maculatus & Anisakidae & N. Java & Burhanuddin \& Djamali (1983) & \\
\hline E. ongus & A. $t$. indonesiensis & N. Java & Present study & Yes \\
\hline $\begin{array}{l}\text { E. quoianus } \\
(=\text { E. megachir })^{\mathrm{a}}\end{array}$ & Anisakidae & N. Java, Sulawesi & Burhanuddin \& Djamali (1983) & \\
\hline E. sexfasciatus & A. t. indonesiensis & N. Java & Present study & Yes \\
\hline $\begin{array}{l}\text { E. summana } \\
(\sim \text { E. ongus or } \\
\text { E. coeruleopunctatus })^{\mathrm{c}}\end{array}$ & Anisakidae & N. Java & Burhanuddin \& Djamali (1983) & \\
\hline
\end{tabular}


Table 3 (continued)

\begin{tabular}{|c|c|c|c|c|}
\hline $\begin{array}{l}\text { Fish host: } \\
\text { family and species }\end{array}$ & Parasite & Locality & Reference & $\begin{array}{l}\text { DNA } \\
\text { sequenced }\end{array}$ \\
\hline Plectropomus leopardus & Anisakis sp. & Bali, Sulawesi & $\begin{array}{l}\text { Asmanelli et al. (1993), } \\
\text { Koesharyani et al. (2001) }\end{array}$ & \\
\hline \multicolumn{5}{|l|}{ Gempylidae } \\
\hline Gempylus serpens & Anisakis sp. & S. Java & Jakob \& Palm (2006) & \\
\hline \multicolumn{5}{|l|}{ Gerreidae } \\
\hline $\begin{array}{l}\text { Gerres oblongus } \\
\text { Haemulidae }\end{array}$ & A. t. indonesiensis & Papua New Guinea & Koinari et al. (2013) & Yes \\
\hline $\begin{array}{l}\text { Pomadasys maculatus } \\
\text { Leiognathidae }\end{array}$ & A. t. indonesiensis & S. Java & Present study & Yes \\
\hline \multicolumn{5}{|l|}{ Lutjanidae } \\
\hline $\begin{array}{l}\text { Lutjanus } \\
\text { argentimaculatus }\end{array}$ & A. $t$. indonesiensis & Bali & Present study & Yes \\
\hline L. kasmira & Anisakidae & N. Java & Ilahude (1980) & \\
\hline L. malabaricus & Anisakis sp. & S. Java & Setyobudi et al. (2011) & \\
\hline L. vitta & A. t. indonesiensis & Bali & Present study & Yes \\
\hline Pinjalo lewisi & A. t. indonesiensis & Papua New Guinea & Koinari et al. (2013) & Yes \\
\hline \multicolumn{5}{|l|}{ Mullidae } \\
\hline \multicolumn{5}{|l|}{ Nemipteridae } \\
\hline \multicolumn{5}{|l|}{ Platycephalidae } \\
\hline 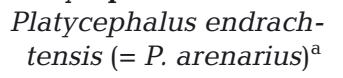 & Anisakis sp. & S. Java & Theisen (2009) & \\
\hline \multicolumn{5}{|l|}{ Priacanthidae } \\
\hline $\begin{array}{l}\text { Priacanthus tayenus } \\
\text { Pristigasteridae }\end{array}$ & A. t. indonesiensis & S. Java & Present study & Yes \\
\hline $\begin{array}{l}\text { Opisthopterus tardoore } \\
\text { Scombridae }\end{array}$ & A. t. indonesiensis & S. Java & Present study & Yes \\
\hline \multirow[t]{6}{*}{ Auxis rochei rochei } & A. typica (s.s.) & 'Indonesia', Bali & $\begin{array}{c}\text { Palm et al. (2008), } \\
\text { Kuhn et al. (2013), present study }\end{array}$ & Yes \\
\hline & A. typica $\times$ A. t. indon & siensis Bali & Palm et al. (2008) & Yes \\
\hline & A. t. indonesiensis & 'Indonesia', Bali & $\begin{array}{c}\text { Palm et al. (2008), } \\
\text { Kuhn et al. (2013), present study }\end{array}$ & Yes \\
\hline & A. pegreffii & Bali & Present study & Yes \\
\hline & A. physeteris & Bali & Present study & Yes \\
\hline & A. berlandi & Bali & Present study & Yes \\
\hline \multirow[t]{2}{*}{ A. thazard thazard } & A. typica (s.s.) & Makassar Strait, Bali & $\begin{array}{l}\text { Anshary et al. (2014), H. Anshary } \\
\text { (pers. comm.), present study }\end{array}$ & Yes \\
\hline & A. t. indonesiensis & $\begin{array}{c}\text { 'Indonesia', Makassar } \\
\text { Strait, Bali }\end{array}$ & $\begin{array}{l}\text { Kuhn et al. (2013), Anshary et al. } \\
\text { (2014), H. Anshary (pers. comm.), } \\
\text { present study }\end{array}$ & Yes \\
\hline \multirow[t]{2}{*}{ Euthynnus affinis } & Anisakis sp. ${ }^{\mathrm{d}}$ & N. Java & $\begin{array}{c}\text { Ilahude et al. (1978), } \\
\text { Burhanuddin \& Djamali (1983) }\end{array}$ & \\
\hline & A. typica (s.l.) $)^{\mathrm{b}}$ & Makassar Strait & $\begin{array}{l}\text { Anshary et al. (2014), } \\
\text { H. Anshary (pers. comm.) }\end{array}$ & \\
\hline Katsuwonus pelamis & A. t. indonesiensis & Makassar Strait & $\begin{array}{l}\text { Anshary et al. (2014), } \\
\text { H. Anshary (pers. comm.) }\end{array}$ & Yes \\
\hline Rastrelliger brachysoma & Anisakis sp. & N. Java & Ilahude et al. (1978), Ilahude (1980) & \\
\hline R. kanagurta & A. typica (s.l.) ${ }^{\mathrm{b}}$ & Makassar Strait & $\begin{array}{c}\text { Hadidjaja et al. (1978), Hutomo et al. (19 } \\
\text { Martosewojo (1980), Burhanuddin \& } \\
\text { Djamali (1983) } \\
\text { Anshary et al. (2014), } \\
\text { H. Anshary (pers. comm.) }\end{array}$ & $\begin{array}{l}978), \\
\&\end{array}$ \\
\hline $\begin{array}{l}\text { Scomberomorus } \\
\text { commerson }\end{array}$ & $\begin{array}{l}\text { Anisakidae } \mathrm{d}_{\mathrm{d}, \mathrm{e}} \\
\text { A. } \text { simplex }^{\mathrm{e}}\end{array}$ & $\begin{array}{l}\text { N. Java } \\
\text { Kupang }\end{array}$ & $\begin{array}{c}\text { Burhanuddin \& Djamali (1983) } \\
\text { Lester et al. (2001) }\end{array}$ & \\
\hline
\end{tabular}


Table 4. Molecular identity and distribution of Anisakis spp. (in \% and absolute numbers) from Indonesian waters with teleost host and ecology (118 of 243 worms from present study, 125 worms from previous studies; see Table 3). Habitat data from Froese \& Pauly (2016)

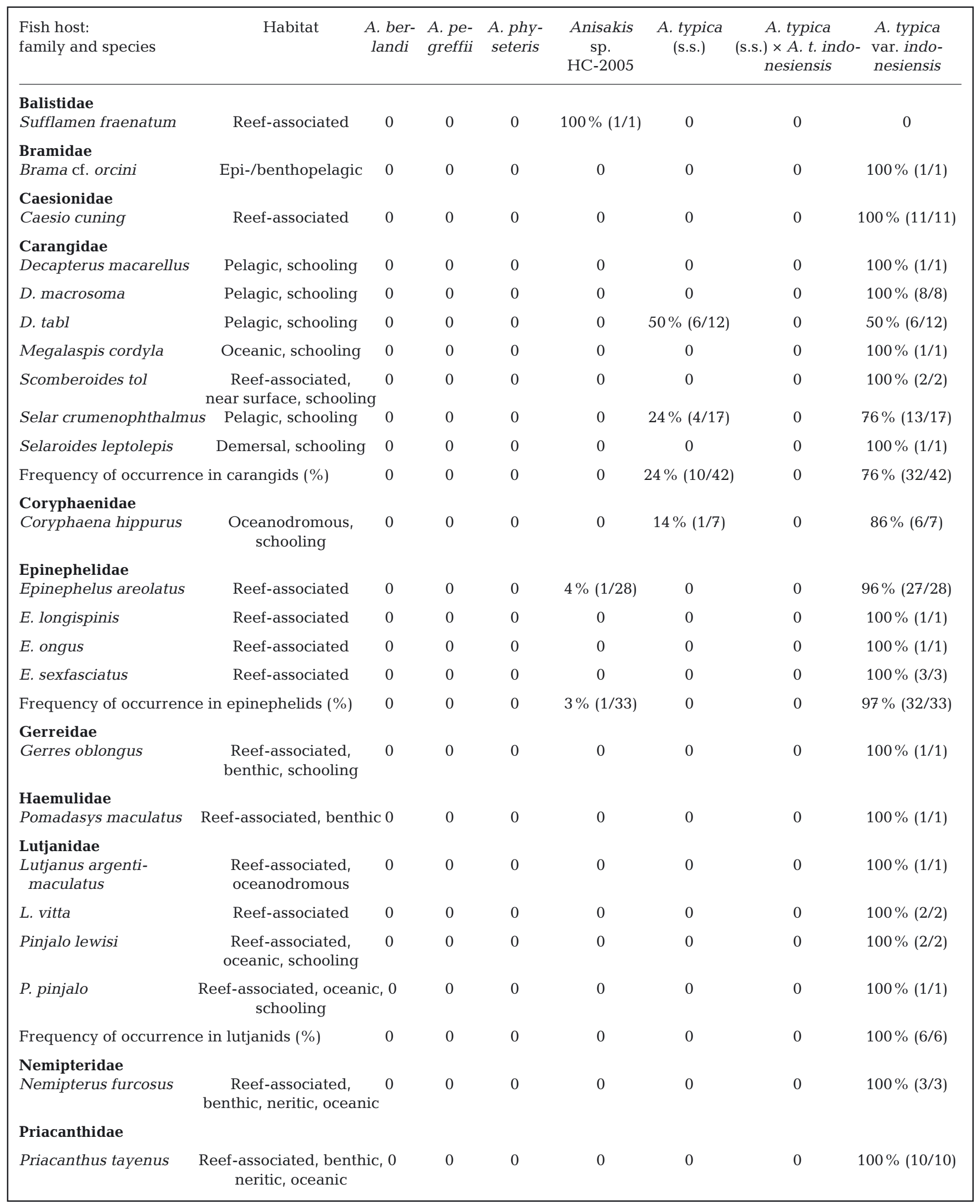


Table 4 (continued)

\begin{tabular}{|c|c|c|c|c|c|c|c|c|}
\hline $\begin{array}{l}\text { Fish host: } \\
\text { family and species }\end{array}$ & Habitat & $\begin{array}{l}\text { A. ber- } \\
\text { landi }\end{array}$ & $\begin{array}{l}\text { A. pe- } \\
\text { greffii }\end{array}$ & $\begin{array}{l}\text { A. phy- } \\
\text { seteris }\end{array}$ & $\begin{array}{l}\text { Anisakis } \\
\text { sp. } \\
\text { HC-2005 }\end{array}$ & $\begin{array}{l}\text { A. typica } \\
\text { (s.s.) }\end{array}$ & $\begin{array}{c}\text { A. typica } \\
\text { (s.s.) } \times \text { A.t. indo- } \\
\text { nesiensis }\end{array}$ & $\begin{array}{l}\text { A. typica } \\
\text { var. indo- } \\
\text { nesiensis }\end{array}$ \\
\hline \multicolumn{9}{|l|}{ Pristigasteridae } \\
\hline Opisthopterus tardoore & $\begin{array}{l}\text { Pelagic, neritic, } \\
\text { oceanodromous }\end{array}$ & 0 & 0 & 0 & 0 & 0 & 0 & $100 \%(2 / 2)$ \\
\hline \multicolumn{9}{|l|}{ Scombridae } \\
\hline Auxis rochei & $\begin{array}{l}\text { Pelagic, oceanodro- } \\
\text { mous, schooling }\end{array}$ & $\begin{array}{l}-\quad 4 \% \\
(2 / 46)\end{array}$ & $\begin{array}{c}2 \% \\
(1 / 46)\end{array}$ & $\begin{array}{c}9 \% \\
(4 / 46)\end{array}$ & 0 & $\begin{array}{c}24 \% \\
(11 / 46)\end{array}$ & $2 \%(1 / 46)$ & $59 \%(27 / 46)$ \\
\hline A. thazard & $\begin{array}{l}\text { Pelagic, oceanodro- } \\
\text { mous, schooling }\end{array}$ & - 0 & 0 & 0 & 0 & $23 \%(7 / 30)$ & 0 & $77 \%(23 / 30)$ \\
\hline Katsuwonus pelamis & $\begin{array}{l}\text { Pelagic, oceanodro- } \\
\text { mous, schooling }\end{array}$ & 0 & 0 & 0 & 0 & $100 \%(5 / 5)$ & 0 & 0 \\
\hline $\begin{array}{l}\text { Scomberomorus } \\
\text { maculatus }\end{array}$ & $\begin{array}{l}\text { Pelagic, oceanodro- } \\
\text { mous, schooling }\end{array}$ & 0 & 0 & 0 & 0 & $100 \%(1 / 1)$ & 0 & 0 \\
\hline Thunnus albacares & $\begin{array}{l}\text { Pelagic, oceanodro- } \\
\text { mous, schooling }\end{array}$ & 0 & 0 & 0 & 0 & $100 \%(1 / 1)$ & 0 & 0 \\
\hline \multicolumn{2}{|c|}{ Frequency of occurrence in scombrids (\%) } & $\begin{array}{c}2.4 \% \\
(2 / 83)\end{array}$ & $\begin{array}{c}1.2 \% \\
(1 / 83)\end{array}$ & $\begin{array}{l}4.8 \% \\
(4 / 83)\end{array}$ & 0 & $\begin{array}{l}30.1 \% \\
(25 / 83)\end{array}$ & $1.2 \%(1 / 83) 6$ & $60.2 \%(50 / 83)$ \\
\hline \multicolumn{9}{|l|}{ Terapontidae } \\
\hline Terapon jarbua & Demersal, schooling & $g \quad 0$ & 0 & 0 & 0 & 0 & 0 & $100 \%(12 / 12)$ \\
\hline \multicolumn{9}{|l|}{ Trichiuridae } \\
\hline Lepturacanthus savala & Benthopelagic & 0 & 0 & 0 & $33 \%(1 / 3)$ & 0 & 0 & $66 \%(2 / 3)$ \\
\hline Trichiurus lepturus & Benthopelagic & 0 & 0 & 0 & 0 & $11 \%(3 / 27)$ & 0 & $89 \%(24 / 27)$ \\
\hline \multicolumn{2}{|c|}{ Frequency of occurrence in trichiurids (\%) } & 0 & 0 & 0 & $3 \%(1 / 30)$ & $10 \%(3 / 30)$ & 0 & $87 \%(26 / 30)$ \\
\hline \multicolumn{2}{|c|}{ Frequency of occurrence in total (\%) } & $\begin{array}{c}0.8 \% \\
(2 / 243)\end{array}$ & $\begin{array}{c}0.4 \% \\
(1 / 243)\end{array}$ & $\begin{array}{c}1.7 \% \\
(4 / 243)\end{array}$ & $\begin{array}{c}1.2 \% \\
(3 / 243)\end{array}$ & $\begin{array}{c}16.1 \% \\
(39 / 243)\end{array}$ & \multicolumn{2}{|c|}{$\begin{array}{c}0.4 \% \\
(1 / 243)\end{array}$} \\
\hline
\end{tabular}

a wide range of (bentho)pelagic, oceanodromous and usually schooling, migratory as well as reef-associated and even demersal fish species. Besides single fish species of the respective families, we also analysed the sequences of pooled Anisakis from 7 different carangids (A. typica: $23.8 \%$; A. typica var. indonesiensis: $76.2 \%$ ), 4 lutjanids (A. typica var. indonesiensis: 100\%), 5 scombrids (A. typica: $30.1 \%$; A. typica var. indonesiensis: $60.2 \%$ ), 3 groupers (epinephelids) (A. typica var. indonesiensis: $97 \%$ ) and 2 trichiurids (A. typica: $7.1 \%$; A. typica var. indonesiensis: 90.5\%) (Table 4).

\section{DISCUSSION}

The present study provides a comprehensive screening and molecular identification of Anisakis spp. from Indonesian waters. Establishing 16 new host records, 53 teleost species are known to be infected with Anisakis in Indonesia, demonstrating the low host-specificity and wide distribution of these taxa.

\section{Hosts and life cycle}

According to Kuhn et al. (2013), the most common teleosts for Anisakis larvae are perciform (57 species) and gadiform (21) species belonging to the fish families Scombridae (12), Gadidae (10), Carangidae (8) and Clupeidae (7), depending on locality and depth of study in the respective region. Abollo et al. (2001) and Klimpel et al. (2004) reported 200 fish species worldwide to be infected with Anisakis larvae, 155 of 
them with genetic evidence (Kuhn et al. 2013). Since then, new collections with genetically identified worms have originated from teleosts already stated in Kuhn et al. (2013) (Anshary et al. 2014, Bak et al. 2014, Mladineo \& Poljak 2014, Chen \& Shih 2015, Cipriani et al. 2015), or from mammalian definitive hosts (i.e. Shamsi 2014, Blažeković et al. 2015). With 53 Anisakis-hosting fish species presented in this study, about one-quarter of the worldwide known Anisakis fish host species and one-third of the worldwide hosts of genetically identified Anisakis genotypes have been reported from Indonesia, even though this country is comparatively understudied, considering its high biodiversity. Sixteen of 40 thoroughly sampled fish species (high-amount fish samples during this study) were infected in this present study, so $40 \%$ harboured Anisakis spp. Indonesia is one of the most diverse marine regions in the world, with 3600 marine teleost species (Froese \& Pauly 2016). It can be expected that many further host records for Anisakis spp. will occur in future samplings from the region, because we even recorded Anisakis from the low-amount fish samples during the workshop, demonstrating the low host-specificity in the region (see Tables 1-4).

Of the 6 different genetically identified Anisakis genotypes, 4 of them were identified to species level. A total of $16.1 \%$ of the samples, infecting $9 / 32$ host species, belonged to A. typica (s.s.) (Table 4), a species known from many tropical regions throughout the world. Palm et al. (2008) have already reported this species in teleosts from Indonesia, especially in Coryphaena hippurus and Auxis rochei rochei. The larvae have been found in scombrids and carangids such as Auxis thazard thazard and Thunnus thynnus from Brazil (SW Atlantic); Scomber japonicus and Trachurus picturatus from the NE Atlantic off Madeira; Euthynnus affinis, Scomberomorus commerson, Sarda orientalis and C. hippurus from the west Indian Ocean off Somalia; and from Merluccius merluccius from the eastern Mediterranean Sea (Palm et al. 2008). Further records were provided by Mattiucci et al. (2005) from Florida, Farjallah et al. (2008) from the Mediterranean coast of North Africa (Scomber scombrus, M. merluccius, Phycis phycis), from Australia by Cannon (1977) and more recently by Shamsi $(2007,2014)$ and Jabbar et al. (2012) from the Great Barrier Reef, and by Chen \& Shih (2015) from Taiwan. Kuhn et al. (2013) have already summarized the worldwide known hosts for genetically confirmed worms, naming 26 different teleost species. A. typica is a common parasite of various dolphin species from warmer temperate and tropical waters, belonging to the families Delphinidae, Phocoenidae and Pontoporiidae (see Mattiucci et al. 2002, Kleinertz et al. 2014a). The eggs of the congener A. simplex (s.s.) are expelled from their hosts with the faeces and embryonate in seawater (Klimpel \& Palm 2011). Larvae hatch as free-living third-stage larvae (L3), still surrounded by the sheath of the second-stage larvae (L2), and get eaten by small crustaceans (copepods, euphausiids) as first intermediate hosts. The L3 develops inside the first intermediate host, and larger invertebrates, cephalopods and various fish species serve as transport hosts that acquire the nematodes through the food chain. They can be transferred further into larger transport hosts without moult, acquiring high numbers in these hosts (Jakob \& Palm 2006). The life cycle is completed when the definitive hosts preys upon infected crustaceans, cephalopods or fishes (Kellermanns et al. 2007). The stomach of the studied Auxis rochei rochei by Palm et al. (2008) was filled with small crustaceans and only a few smallsized fish, and Decapterus russelli as a common host preyed upon small crustaceans as well. A low intensity of anisakids in a sampled fish species suggests that the larvae uptake originates directly from the crustacean first intermediate hosts (see Palm 1999), while larger predatory fish also serve as transport host and accumulate the larvae (Table 4). This suggests that several reef fish within the present study, such as Sufflamen fraenatum, accidentally aquire the worms, while the heavily infected pelagic scombrids, carangids and coryphaenids either are infected directly through the first intermediate or serve as transport host, suggesting a pelagic life cycle for the tropical A. typica, as suggested by Palm et al. (2008) and tentatively by Kuhn et al. (2013).

\section{Anisakis typica var. indonesiensis}

The predominant recorded genotype within the present study was A. typica var. indonesiensis, a genotype for the first time reported from Auxis rochei rochei, Caesio cuning, Coryphaena hippurus and Epinephelus areolatus from Indonesia by Palm et al. (2008). It was recently described also from Papua New Guinea (Koinari et al. 2013), Sulawesi, Java and Bali, Indonesia (Kuhn et al. 2013, Anshary et al. 2014, Kleinertz et al. 2014a) under different names, having a distinct $4 \mathrm{bp}$ difference (the same range as recorded for the siblings $A$. simplex (s.s.) and A. berlandi as well as A. pegreffii). Multiple marker studies combined with morphological investigation of the adults must test whether this difference distinguishes a dis- 
tinct species or is a matter of intraspecific variability. Therefore, we refrain from nominating a distinct taxon, but apply the subspecific entity A. typica var. indonesiensis. This genotype seems to be more widely spread than known so far, appearing to have no clear host specificity in Indonesian waters, infecting over 28 (of 32 with genetically identified Anisakis spp. infection) teleost host species belonging to 14 (of 15) families. In contrast, only 6 of these 32 infected Indonesian fish species harbour both the common A. typica (s.s.) as well as this genotype. A single case of an additional genotype reported by Palm et al. (2008) in Auxis rochei, possibly a hybrid between the 2 A. typica genotypes, suggests that both have the same final hosts in the region and are able to infect the same or a similar intermediate host range. Interestingly, Shamsi (2007) also suggested that the A. typica in Australia seems to be genetically different from those reported in other countries, and the cluster analysis of A. typica by Iniguez et al. (2011) showed a cluster consisting only of intermediate/ paratenic hosts from Asian coasts. Adult specimens must be sampled from the final hosts in the region for a future species description, to morphologically distinguish A. typica var. indonesiensis from A. typica (s.s.).

\section{Distribution of Anisakis spp.}

A. berlandi and $A$. pegreffii are reported for the first time from an equatorial region (both have been already reported from Australian mammals, see Shamsi et al. 2012), and A. physeteris is recorded for the first time from the Pacific Ocean. These 3 species were exclusively found in the pelagic, oceanodromous and migratory carangid Auxis rochei. According to Klimpel et al. (2008), Mattiucci \& Nascetti (2007, 2008) and Kellermanns (2009), A. berlandi and A. pegreffii together with A. simplex (s.s.) form the A. simplex s.l. complex of species that are most common in temperate to cold regions, e.g. from $30-70^{\circ} \mathrm{N}$ and S latitudes. A. berlandi so far has been reported from the North and South Pacific, the Atlantic coast of southern Africa, and from Australia and off New Zealand (Mattiucci et al. 1997, 2014, Mattiucci \& Nascetti 2006, 2007, 2008, Klimpel et al. 2008, Kellermanns 2009), and has been found in the migrating pilot whale Globicephala melas (Mattiucci et al. 2014) as an adult. A. pegreffii infects delphinids, ziphiids, physeterids and neobalaenids as final hosts and has been reported from the waters off Italy, Argentina, Brazil, South Africa and New Zealand
(Klimpel et al. 2008, Mattiucci \& Nascetti 2008, Kellermanns 2009), especially in the temperate zones. Recent cases of anisakiasis caused by A. pegreffii in Korea (Lim et al. 2015), Italy and Japan (Mattiucci et al. 2013, Lim et al. 2015) demonstrate that besides $A$. simplex (s.s.), A. pegreffii can also become human-pathogenic. Interestingly, A. simplex (s.s.), commonly found in the northern hemisphere and also in Japan in the North Pacific, could not be identified within the present study. This is of importance because $A$. simplex is the main species causing anisakiasis. Also A. physeteris, infecting physeterid whales as adults, has been reported from temperate regions, e.g. the Atlantic and Mediterranean, but not yet from the Pacific Ocean. However, physeterid whales are found worldwide, also in the Pacific. Consequently, the present study sheds some light on the general distribution patterns of Anisakis infections in the world oceans. Though only rare findings of the above species from the low latitudes do exist, they also occur as larvae in teleosts of the tropics, and future findings are expected. Because the migrating Auxis rochei from Indonesia is widely distributed within the region but does not migrate into temperate zones, they must have acquired the Anisakis larvae from the first or second intermediate hosts around Indonesian waters. Whales as final hosts are able to release the eggs while migrating between the northern and southern hemisphere. However, in the case of A.berlandi, A. pegreffii and A. physeteris, the larvae seem to be able to infect also intermediate hosts in tropical waters, explaining their extensive range of distribution. This might be different for $A$. simplex (s.s.) which is restricted to the boreal zone, and especially occurs in the northern hemisphere. The species might have a more restricted first intermediate host regime. Similarly, the genotype Anisakis sp. HC-2005 so far has been reported from 3 different teleost hosts in Indonesia (Sufflamen fraenatum, present study; Lepturacanthus savala, Kuhn et al. 2013; and Epinephelus areolatus, Kleinertz et al. 2014a), and from northwest Africa (Hoplostethus cadenati from the African shelf, see Kijewska et al. 2009), and seems to be not able to extend the host range into the more northern and southern regions. This underlines the importance of low host-specificity to infect a range of first intermediate hosts to extend the host distribution in the anisakid nematodes, as earlier suggested by Kellermanns et al. (2007). The other factors supporting constant gene flow (see Mattiucci et al. 2002) are extensive final and intermediate host migration, overlapping distribution patterns of different final host populations, 
and large population sizes in the intermediate and final hosts (Palm 2004, Kellermanns et al. 2007, Palm et al. 2007).

\section{Infection patterns and site}

Analyses of the infected teleost hosts and their ecology reveal a distinct pattern, with highest infection rates in the scombrids and carangids and lowest in some reef-associated fish (cf. Tables $1 \& 2$ ). We did not expect differences during the rainy versus dry seasons, because the worms are quite long-living. The fact that $94 \%$ of the worms were sampled throughout the dry seasons is a result of uneven distribution in the fishes, because 667 worms (of all 848) were isolated from a sample of 36 Auxis rochei during the dry season only (cf. Tables $1 \& 2$ ). The observed prevalence and the intensity of infection of Anisakis spp. were highest in the pelagic schooling scombrid Auxis rochei, also getting infected with 5 of the 6 detected Anisakis taxa. This leads to the as. sumption that this fish species is the most suitable intermediate host for Anisakis nematodes in the sampled region. A. typica (s.l.) infections of Auxis rochei rochei are readily observed throughout the years, especially between 2005 and 2006 (Palm et al. 2008) and 2013 (present study), with a minimum prevalence of $20 \%$. This complies with the presence of the potential final hosts in the region. Other high infection rates within this study were observed in the oceanic/pelagic/schooling Auxis thazard, Selar crumenophthalmus and Decapterus tabl, the benthopelagic Trichiurus lepturus and also in the more reefassociated Epinephelus areolatus and Caesio cuning (cf. Tables 2-4) and Priacanthus tayenus. This might refer to the distribution of the dolphin final hosts in the region that migrate throughout the archipelago but also close to coral reef habitats, and may also feed on schooling demersal fish over shallow sandy bottoms (compared to the high prevalence of infection in Terapon jarbua). Consequently, the occurrence in reef-associated fish does not refer to a benthic parasite life cycle but to the wide distribution of the suitable first intermediate hosts (offshore to close to the reef ecosystems), low host-specificity concerning the teleost hosts (hundreds of possible intermediate fish host species), and to the mobility of the dolphin final hosts between the different tropical habitats and food webs. This possibly overcomes the limitations of the otherwise highly specialized food web in coral reef habitats, enabling Anisakis to infect such a wide host range.
The typical infection site for Indonesian Anisakis spp. was the body cavity, with $\mathrm{n}=131$ free-living worms, and others attached to the mesenteries and outer surfaces of the liver $(\mathrm{n}=299)$, gonads $(\mathrm{n}=$ 138), stomach $(\mathrm{n}=136)$ and guts (intestine, $\mathrm{n}=95$; pyloric caeca, $\mathrm{n}=45$ ). Because most of the recorded Anisakis belonged to A. typica and A. typica var. indonesiensis, these are the preferred infection sites for these 2 genotypes. Previous studies reported no muscular infections (Burhanuddin \& Djamali 1978, 1983, Ilahude et al. 1978), Palm et al. (2008) found only a single case in 110 examined Auxis rochei, Anshary et al. (2014) stated possible migration into the musculature, and the present study documented a single specimen from the musculature of Selar crumenophthalmus. In contrast, the related A. simplex (s.s.) that is known as a main cause of zoonotic anisakiasis often infects fish musculature in temperate waters (e.g. Strømnes \& Andersen 1998, 2003). Palm et et al. (2008) suggested that besides the life cycle and dispersal mechanism, a typical site of infection might be another distinguishing feature among the different Anisakis taxa. This can explain why A. typica (s.1.) so far has not been affiliated with human anisakiasis, and no case of anisakiasis from Bali has been recorded (also see Palm et al. 2008). However, this contradicts Uga et al. (1996), who found Anisakis antibodies in a number of Javanese people. The only explanation is that though the most common A. typica (s.l.) are regularly taken up and become digested by consumers, they seem to cause no major infection with serious disease symptoms. This suggests a general low risk of Indonesian fisheries consumers to develop anisakiasis. While the most dangerous A. simplex (s.s.) could not be recorded from Indonesia, we isolated only a single A. pegreffii from an Auxis rochei, which is nowadays known to cause anisakiasis (3 cases worldwide). However, it is not clear which Anisakis species caused the immunological reaction identified by Uga et al. (1996).

\section{Outlook}

There are 3 main outcomes for future studies. First, we recommend sampling adults of A. typica var. indonesiensis for a morphological species description in future. This can be done either by dissecting stranded marine mammals or via an underwater sampling while diving with dolphins, wild or trained for animal health monitoring purposes. Kleinertz et al. (2014b) demonstrated the functionality of the lat- 
ter. Second, until a new species description and taxonomical treatment has been completed, we strongly recommend the name A. typica var. indonesiensis when dealing with this Indonesian taxon or its genotype, because this genotype has been documented as the predominant one in Indonesia. It also cannot be excluded that it will be reported from other tropical regions outside Indonesia in future. Third, taking into account various Anisakis species in a broad range of fish host species, the transfer of northern and southern species via migratory hosts into the region, and positive tests for Anisakis antibodies of Indonesian citizens as well as the documentation of the human-pathogenic species A. pegreffii, we suggest seroepidemiological or stereoscopical tests of Indonesian citizens. Although presumably at a low risk, additional insights into the real anisakiasis impact in Indonesia, the 4 th most populous nation of the world with almost every citizen reliant on ocean and fisheries supplies, but also with a low standard in the medical system, are still required. Consumers should thus be informed that the scombrid Auxis rochei bears the highest risk of anisakiasis infection in Indonesia, and should not be used in dishes using raw or semi-cooked fish.

Acknowledgements. This study was presented as a poster at the 9th International Symposium on Fish Parasites, Valencia, 31 August to 4 September 2015. The study was supported through a $\mathrm{PhD}$ scholarship programme provided by the German Academic Exchange Service (DAAD, budgetary title 332404 101, code digit D/09/48018) (S.T.) and the Indonesian HIBAH competitive grant (H.W.P., I.M.D., N.A.S., I.B.M.O., S.K.). Further samplings were made within the joint Indonesian-German research programme SPICE III-MABICO (Science for the Protection of Indonesian Coastal Marine Ecosystems, BMBF grant no. 03F0641D) (S.K., H.W.P.), especially during the First Educational Workshop on Marine Fish Parasites in Indonesia, 21 July to 2 August 2013, Denpasar, Bali. We are thankful to Dr. Irfan Yulianto for the fish sampling from Karimunjawa National Park, permitted by the National Park authority (approval no. 18/BA/BTNKJ-3/2013). We are also thankful to the Indonesian (former) bachelor students Faiza Nurul Falacy, I Gusti Agung Made Armada Hambarsika, Rezki Alifia Hartono, Fajar Diyaa'ul Iman, Escha Firdaus Kayuzi, Challa Victora Ndun, Maria Anisiata Ningsi, I Putu Gede Hendra Pradipta, Maulida Nur Rahmawati, Berna Natalia Silaban, Mori Friska Tamba, Toni Irawan Wibisomo, Oscar Yudistira and Afif Naufal Zainurrahman from Udayana and UNSOED universities for their support during sampling and laboratory work. This is publication no. 7 under the Memorandum of Understanding between the Faculty of Veterinary Medicine, Udayana University, Bali, and the Faculty of Agricultural and Environmental Sciences, Aquaculture and Sea-Ranching, University of Rostock, Germany, in order to promote fish parasite and biodiversity research in Indonesia.

\section{LITERATURE CITED}

Abollo E, Gestal C, Pascual S (2001) Anisakis infestation in marine fish and cephalopods from Galician waters: an updated perspective. Parasitol Res 87:492-499

Anderson RC (2000) Nematode parasites of vertebrates. Their development and transmission, 2nd edn. CABI Publishing International, Wallingford

Anderson RC, Chabaud AG, Willmott S (2009) Keys to the nematode parasites of vetebrates-archival volume. $\mathrm{CAB}$ International, Wallingford

* Anshary H, Sriwula, Freeman MA, Ogawa K (2014) Occurrence and molecular identification of Anisakis Dujardin, 1845 from marine fish in Southern Makassar Strait, Indonesia. Korean J Parasitol 52:9-19

Asmanelli, Yuliansyah H, Muchari (1993) Penyakit ikan laut di lokasi Keramba Jaring Apung di Kepulauan Riau [Marine fish diseases in floating net cages in Riau Archipelago]. Prosiding Seminar hasil penelitian perikanan budidaya pantai, 16-19 Juli 1993, Maros, Indonesia, Vol 11, p 13-24 (in Indonesian)

Bak TJ, Jeon CH, Kim JH (2014) Occurrence of anisakid nematode larvae in chub mackerel (Scomber japonicus) caught off Korea. Int J Food Microbiol 191:149-156

* Blažeković K, Pleić IL, Duras M, Gomerčić T, Mladineo I (2015) Three Anisakis spp. isolated from toothed whales stranded along the eastern Adriatic Sea coast. Int J Parasitol 45:17-31

Burhanuddin, Djamali A (1978) Parasit Anisakis sebagai petunjuk perbedaan populasi ikan laying, Decapterus russelli Ruppell, di laut Jawa. Oseanol Indones 9:1-11 (in Indonesian)

Burhanuddin, Djamali A (1983) Pengamatan larva Anisakidae pada ikan laut di laut Jawa dan sekitarnya. Oseanol Indones 16:19-27 (in Indonesian)

* Bush AO, Lafferty KD, Lotz JM, Shostak AW (1997) Parasitology meets ecology on its own terms: Margolis et al. revisited. J Parasitol 83:575-583

Cannon LRG (1977) Some aspects of the biology of Peponocephala electra (Cetacea: Delphinidae). II. Parasites. Aust J Mar Freshw Res 28:717-722

* Chen HY, Shih HH (2015) Occurrence and prevalence of fish-borne Anisakis larvae in the spotted mackerel Scomber australasicus from Taiwanese waters. Acta Trop 145:61-67

* Cipriani P, Smaldone G, Acerra V, D'Angelo L and others (2015) Genetic identification and distribution of the parasitic larvae of Anisakis pegreffii and Anisakis simplex (s. s.) in European hake Merluccius merluccius from the Tyrrhenian Sea and Spanish Atlantic coast: implications for food safety. Int J Food Microbiol 198:1-8

F Farjallah S, Ben Slimane B, Busi M, Paggi L and others (2008) Occurrence and molecular identification of Anisakis spp. from the North African coasts of Mediterranean Sea. Parasitol Res 102:371-379

Fischer W, Whitehead PJP (eds) (1974) FAO species identification sheets for fishery purposes, Vols 1-4. Eastern Indian Ocean (fishing area 57) and Western Central Pacific (fishing area 71). FAO, Rome

Froese R, Pauly D (eds) (2016) FishBase. www.fishbase.org (accessed 01/2016)

พ Hadidjaja P, Ilahude H, Mahfudin B, Malikusworo H (1978) Larvae of Anisakidae in marine fish of coastal waters near Jakarta, Indonesia. Am J Trop Med Hyg 27:51-54

Heemstra PC, Randall JE (1993) FAO Species Catalogue, 
Vol 16. Groupers of the world (family Serranidae, subfamily Epinephelinae). An annotated and illustrated catalogue of the grouper, rockcod, hind, coral grouper and lyretail species known to date. FAO Fish Synop 125(16). FAO, Rome

Hutomo M, Burhanuddin, Hadidjaja P (1978) Observations on the incidence and intensity of infection of nematode larvae (Fam. Anisakidae) in certain marine fishes of waters around Panggang Island, Seribu Islands. Mar Res Indones 21:49-60

ICZN (International Commission on Zoological Nomenclature) (2016) International Code of Zoological Nomenclature, 4th edn. www.nhm.ac.uk/hosted-sites/iczn/code/ index.jsp (accessed 11/2016)

Ilahude HD (1980) Anisakid larvae in marine fish in Indonesia (a review). Asian Meeting on Parasitic Infections, Feb 26-28, Bangkok

Ilahude HD, Hadidjaja P, Mahfudin B (1978) Survey on anisakid larvae in marine fish from markets in Jakarta. Southeast Asian J Trop Med Public Health 9:48-50

Iniguez AM, Carvalho VL, Alves Motta MR, Sousa Nunes Pinheiro DC, Paulo Vicente AC (2011) Genetic analysis of Anisakis typica (Nematoda: Anisakidae) from cetaceans of the northeast coast of Brazil: new data on its definitive hosts. Vet Parasitol 178:293-299

Ishikura H, Kikuchi K (1990) Intestinal anisakiasis in Japan. Springer, Tokyo

Ishikura H, Namiki M (1989) Gastric anisakiasis in Japan. Springer, Tokyo

Ishiwata K, Shinohara A, Yagi K, Horii Y, Tsuchiya K, Nawa Y (2004) Identification of tissue-embedded ascarid larvae by ribosomal DNA sequencing. Parasitol Res 92:50-52

Ivanovic J, Baltic MZ, Boskovic M, Kilibarda N and others (2015) Anisakis infection and allergy in humans. Procedia Food Sci 5:101-104

Jabbar A, Asnoussi A, Norbury LJ, Eisenbarth A and others (2012) Larval anisakid nematodes in teleost fishes from Lizard Island, northern Great Barrier Reef, Australia. Mar Freshw Res 63:1283-1299

Jakob E, Palm HW (2006) Parasites of commercially important fish species from the southern Java coast, Indonesia, including the distribution pattern of trypanorhynch cestodes. Verh Ges Ichthyol 5:165-191

Kellermanns E (2009) Fischparasiten der bathydemersalen Zone: Charakterisierung, Verbreitungsmuster und Lebenszyklen. PhD thesis, University of Düsseldorf

Kellermanns E, Klimpel S, Palm HW (2007) Molecular identification of ascaridoid nematodes from the deep-sea onion-eye grenadier (Macrourus berglax) from the East Greenland Sea. Deep-Sea Res I 54:2194-2202

Kijewska A, Dzido J, Rokicki J (2009) Anisakid parasites of fishes caught on the African shelf. J Parasitol 95:639-645

Kleinertz S, Damriyasa IM, Hagen W, Theisen S, Palm HW (2014a) An environmental assessment of the parasite fauna of the reef-associated grouper Epinephelus areolatus from Indonesian waters. J Helminthol 88:50-63

Kleinertz S, Hermosilla C, Ziltener A, Hirzmann J, AbdelGhaffar F, Taubert A (2014b) Gastrointestinal parasites of free-living Indo-Pacific bottlenose dolphins (Tursiops aduncus) in the Northern Red Sea, Egypt. Parasitol Res 113:1405-1415

Klimpel S, Palm HW (2011) Anisakid nematode (Ascaridoidea) life cycles and distribution: increasing zoonotic potential in the time of climate change? In: Mehlhorn $\mathrm{H}$ (ed) Progress in parasitology. Parasitology Research
Monographs, Vol 2. Springer Verlag, Berlin, p 201-222

K Klimpel S, Palm HW, Rückert S, Piatkowski U (2004) The life cycle of Anisakis simplex in the Norwegian Deep (northern North Sea). Parasitol Res 94:1-9

Klimpel S, Kellermanns E, Palm HW (2008) The role of pelagic swarm fish (Myctophidae: Teleostei) in the oceanic life cycle of Anisakis sibling species at the MidAtlantic Ridge, Central Atlantic. Parasitol Res 104:43-53

Koesharyani I, Roza D, Mahardika K, Johnny F, Zafran, Yuasa K (2001) Manual for fish disease diagnosis. II. Marine fish and crustacean diseases in Indonesia. Gondol Research Institute for Mariculture, Central Research Institute for Sea Exploration and Fisheries, Department of Marine Affairs and Fisheries Singaraja, Bali, and Japan International Cooperation Agency, Tokyo

Koinari M, Karl S, Elliot A, Ryan U, Lymbery AJ (2013) Identification of Anisakis species (Nematoda: Anisakidae) in marine fish hosts from Papua New Guinea. Vet Parasitol 193:126-133

*Kuhn T, García-Màrquez J, Klimpel S (2011) Adaptive radiation within marine anisakid nematodes: a zoogeographical modeling of cosmopolitan, zoonotic parasites. PLOS ONE 6:e28642

Kuhn T, Hailer F, Palm HW, Klimpel S (2013) Global assessment of molecular identified Anisakis spp. (Nematoda: Anisakidae) in the teleost intermediate host. Folia Parasitol 4826:1-29

Lester RJG, Thompson C, Moss H, Barker SC (2001) Movement and stock structure of narrow-barred Spanish mackerel as indicated by parasites. J Fish Biol 59: 833-842

* Lim H, Jung BK, Cho J, Yooyen T, Shin EH, Chai JY (2015) Molecular diagnosis of cause of anisakiasis in humans, South Korea. Emerg Infect Dis 21:342-344

Lymbery AJ, Cheah FY (2007) Anisakid nematodes and anisakiasis. In: Murrell KD, Fried B (eds) Food borne parasitic zoonosis: fish and plant-borne parasites. Springer, New York, NY, p 185-207

Martosewojo S (1980) Cacing Anisakis yang hidup sebagai parasit pada ikan laut. Pewarta Oseana 6:1-5

*Mattiucci S, Nascetti G (2006) Molecular systematics, phylogeny and ecology of anisakid nematodes of the genus Anisakis Dujardine, 1845: an update. Parasite 13:99-113

Mattiucci S, Nascetti G (2007) Genetic diversity and infection levels of anisakid nematodes parasitic in fish and marine mammals from Boreal and Austral hemispheres. Vet Parasitol 148:43-57

Mattiucci S, Nascetti G (2008) Advances and trends in the molecular systematics of anisakid nematodes, with implications for their evolutionary ecology and host-parasite co-evolutionary processes. Adv Parasitol 66:47-148

Mattiucci S, Nascetti G, Cianchi R, Paggi L and others (1997) Genetic and ecological data on the Anisakis simplex complex, with evidence for a new species (Nematoda, Ascaridoidea, Anisakidae). J Parasitol 83:401-416

*Mattiucci S, Paggi L, Nascetti G, Portes Santos C and others (2002) Genetic markers in the study of Anisakis typica (Diesing, 1860): larval identification and genetic relationships with other species of Anisakis Dujardin, 1845 (Nematoda: Anisakidae). Syst Parasitol 51:159-170

Mattiucci S, Nascetti G, Dailey M, Webb SC, Barros NB, Cianchi R, Bullini L (2005) Evidence for a new species of Anisakis (Dujardin, 1845): morphological description and genetic relationships between congeners (Nematoda: Anisakidae). Syst Parasitol 61:157-171 
Mattiucci S, Paoletti M, Webb SC (2009) Anisakis nascettii n. sp. (Nematoda: Anisakidae) from beaked whales of the southern hemisphere: morphological description, genetic relationships between congeners and ecological data. Syst Parasitol 74:199-217

Mattiucci S, Fazii P, De Rosa A, Paoletti M and others (2013) Anisakiasis and gastroallergic reactions associated to Anisakis pegreffii infection, Italy. Emerg Infect Dis 19:496-499

Mattiucci S, Cipriani P, Webb SC, Paoletti M, Marcer F, Bellisario Gibson DI, Nascetti G (2014) Genetic and morphological approaches distinguish the three sibling species of the Anisakis simplex species complex, with a species designation as Anisakis berlandi n. sp. for A. simplex sp. C (Nematoda: Anisakidae). J Parasitol 100:199-214

Mladineo I, Poljak V (2014) Ecology and genetic structure of zoonotic Anisakis spp. from Adriatic commercial fish species. Appl Environ Microbiol 80:1281-1290

Nadler SA, d'Amelio S, Dailey MD, Paggi L, Siu S, Sakanari J (2005) Molecular phylogenetics and diagnosis of Anisakis, Pseudoterranova, and Contracaecum from the northern Pacific marine mammals. J Parasitol 91:1413-1429

Nieuwenhuizen N (2016) Anisakis - immunology of a foodborne parasitosis. Parasite Immunol 38:548-557

* Palm HW (1999) Ecology of Pseudoterranova decipiens (Krabbe, 1878) (Nematoda: Anisakidae) from Antarctic waters. Parasitol Res 85:638-646

Palm HW (2004) The Trypanorhyncha Diesing, 1863. PKSPLIPB Press, Bogor

Palm HW, Bray RA (2014) Fish parasitology in Hawaii. Westarp \& Partner Digitaldruck, Hohenwarsleben

* Palm HW, Waeschenbach A, Littlewood DTJ (2007) Genetic diversity in the trypanorhynch cestode Tentacularia coryphaenae Bosc, 1797: evidence for a cosmopolitan distribution and low host specificity in the teleost intermediate host. Parasitol Res 101:153-159

Palm HW, Damriyasa IM, Linda Oka IBM (2008) Molecular genotyping of Anisakis Dujardin, 1845 (Nematoda: Ascaridoidea: Anisakidae) larvae from marine fish of Balinese and Javanese waters, Indonesia. Helminthologia 45:3-12

Petersen F, Palm H, Möller H, Cuzi MA (1993) Flesh parasites of fish from central Philippine waters. Dis Aquat Org 15:81-86

Pontes T, d'Amelio S, Costa G, Paggi L (2005) Molecular characterization of larval anisakid nematodes from marine fishes of Madeira by a PCR-based approach, with evidence for a new species. J Parasitol 91:1430-1434

Setyobudi E, Soeparno, Helmiati S (2011) Infection of Anisakis sp. larvae in some marine fishes from the south-

Editorial responsibility: Sven Klimpel,

Frankfurt, Germany ern coast of Kulon Progo, Yogyakarta. Biodiversitas 12: $34-37$

Shamsi S (2007) Morphologic and genetic characterisation of selected ascaridoid nematodes. University of Melbourne, Melbourne

Shamsi S (2014) Recent advances in our knowledge of Australian anisakid nematodes. Int J Parasitol Parasites Wildl 3:178-187

Khamsi S, Gasser R, Beveridge I (2012) Genetic characterisation and taxonomy of species of Anisakis (Nematoda: Anisakidae) parasitic in Australian marine mammals. Invertebr Syst 26:204-212

Sohn WM, Na BK, Kim TH, Park TJ (2015) Anisakiasis: report of 15 gastric cases caused by Anisakis type I larvae and a brief review of Korean anisakiasis cases. Korean J Parasitol 53:465-470

* Strømnes E, Andersen K (1998) Distribution of whaleworm (Anisakis simplex, Nematoda, Ascaridoidea) L3 larvae in three species of marine fish; saithe (Pollachius virens (L.)), cod (Gadus morhua L.) and redfish (Sebastes marinus (L.)) from Norwegian waters. Parasitol Res 84:281-285

* Strømnes E, Andersen K (2003) Growth of whaleworm (Anisakis simplex, Nematodes, Ascaridoidea, Anisakidae) third-stage larvae in paratenic fish hosts. Parasitol Res 89:335-341

Theisen S (2009) Fischparasiten von der Südküste Javas, Indonesien. Masters thesis, Mathematisch-Naturwissenschaftliche Fakultät, Heinrich-Heine-Universität Düsseldorf (in German with English abstract)

* Thompson JD, Higgins DG, Gibson TJ (1994) CLUSTAL W: improving the sensitivity of progressive multiple sequence alignment through sequence weighting, positions-specific gap penalties and weight matrix choice. Nucleic Acids Res 22:4673-4680

*Uga S, Ono K, Kataoka N, Hasan H (1996) Seroepidemiology of five major zoonotic parasite infections in inhabitants of Sidoarjo, East Java, Indonesia. Southeast Asian J Trop Med Public Health 27:556-561

*Umehara A, Kawakami Y, Araki J, Uchida A (2008) Multiplex PCR for the identification of Anisakis simplex sensu stricto, Anisakis pegreffii and the other anisakid nematodes. Parasitol Int 57:49-53

Yamaguti S (1954) Parasitic worms mainly from Celebes. Part 9. Nematodes of fishes. Acta Med Okayama 9: 122-133

* Zhu X, d'Amelio S, Paggi L, Grasser RB (2000) Assessing sequence variation in the internal transcribed spacers of ribosomal DNA within and among members of the Contracaecum osculatum complex (Nematoda: Ascaridoidea: Anisakidae). Parasitol Res 86:677-683

Submitted: May 4, 2016; Accepted: December 2, 2016 Proofs received from author(s): February 22, 2017 